International Journal of Solar Thermal Vacuum Engineering

\title{
Dye removal with magnetic graphene nanocomposite through micro reactors
}

\author{
Essam Mohamed Abo-Zahhadd, ${ }^{1}$, A. S. Hammad ${ }^{2}$, Ali Radwan ${ }^{3,4}$, Saim Memon ${ }^{5}$, A. H. El-Shazly ${ }^{3,4}$, \\ M. F. El-Kady ${ }^{6}$ \\ Mechanical Power Engineering Department, Faculty of Energy Engineering, Aswan University, Aswan, Egypt \\ Chemicals and Petrochemicals Engineering Department, Faculty of Engineering Port Said University, Port Said, Egypt \\ 3 Mechanical Power Engineering Department, Faculty of Engineering, Mansoura University, El Mansoura, Egypt \\ 4 Mansoura University Nanotechnology Center, Mansoura University, Mansoura, Egypt \\ 5 Solar Thermal Vacuum Engineering Research Group, London Centre for Energy Engineering, School of Engineering, London South Bank \\ University, London, United Kingdom \\ 6 Chemicals and Petrochemicals Engineering Department, Egypt-Japan University of Science and Technology, New Borg El-Arab City, Alexandria, \\ Egypt
}

\section{ABSTRACT}

Contaminated waste water treatment and clean water scarcity are current challenges acutely in the Asian and African continents. This paper bestows applied co-precipitation technique for the fabrication of Magnetic Graphene Nano-Composites (MGNCs) for water treatment purposes. In this paper, characterization procedures were applied to delineate numerous physical and chemical properties of the synthetic MGNCs and mixing performance for several designed microreactors were determined using the Dushman's method in comparison to two parallel reactions. The mixing timings for different microreactors at flow rates between 100 and $300 \mathrm{ml} / \mathrm{hr}$ were determined. MCNCs were utilised to remove an Acid Blue 25 dye as a pollutant from water at diverse types of microreactors. The comparison between the various microreactors' performance and mixing time was accomplished. The maximum instantaneous removal capacity of graphene-based nanomaterial was recorded using K.M micro mixer about $68 \%$ for 10 ppm dye concentration.

\section{Keywords:}

Magnetic Graphene Nano-Composite;

Water Treatment; Micro Reactors;

decolorization; nanomaterial

Copyright $\odot 2020$ PENERBIT AKADEMIA BARU - All rights reserved

\section{Introduction}

Due to an increase of global population and industrialization causing environmental pollution, primarily affecting the natural water resources. Meanwhile, up to $50 \%$ of worldwide energy needs are derived from the limited fossil fuels resources. Currently, many countries, specifically in the Asian and African continents, are oppressed with clean water scarcity. The freshwater stores present about $2.5 \%$ of worldwide water resources, however just $0.6 \%$ of total natural freshwater are potable and fit the human's uses. Furthermore, freshwater supplies are falling largely because of overexploitation, contamination and climate change. One of the promising solutions to these

\footnotetext{
* Corresponding author.

E-mail address: Essam_Mohamed@aswu.edu.eg
} 
challenges is to make use of waste/recycled water that is available in significant quantities. If all this water could be applied to beneficial uses, recycled/reused water could provide water supply reliability at far less energy than more energy-intensive supply options such as seawater desalination. Therefore, the worldwide market for water and wastewater equipment and services is growing and becoming more competitive due to renewed governments focus on the sector and recently secured investments creating opportunities for foreign and domestic operators and investors. In Egypt, the wastewater treatment plants increase from 149 in 2005 to more than 400 in last year. Such wastewater plants can provide potable water to the 95\% inhabited rural areas in Egypt that is suffering from lack of electricity and fresh water. Wastewater treatment is the process used to treat pollutants and contaminants (i.e., soluble organic matter, suspended solids, pathogenic organisms, etc.) from water streams and convert it into an effluent that can be returned to the water cycle with minimal impact on the environment or directly reused. The significance effect of wastewater treatment comes from polluting the major part of water that can be consumed by human activities. The Egyptian government went up a major program to expand and enhance public infrastructure. As part of this program, the ministry of housing, utilities, and urban development and the construction authority for potable water and wastewater to fund and operate wastewater treatment plants. For instance, the amount of polluted water in petroleum refineries is almost 0.4:1.6 times the amount of processed oil. The removal/treatment techniques to be utilized are classified into physical, chemical, and biological processes. The conventional treatment method of refinery wastewater is based on physicochemical and mechanical methods with further biological treatment within the integrated activated sludge treatment unit. With respect to the fact that different concentrations of aliphatic and aromatic petroleum hydrocarbons are present in the wastewater, among which the aromatic fraction that is more toxic is not readily degraded by conventional treatment, there is still a need for advanced techniques to remove these pollutants.

One of the most dangerous wastes are dyes because of their side effects to human health and the environment $[1,2]$. In addition, the colors of the dyes are simply detectable, even, at a very low level of concentrations, making them visible and unwanted. Enormous methods of dye wastewater treatment such as; anaerobic reduction, adsorption and bacterial decolonization methods have been reviewed by Srinivasan et al.[1], Mondal [2] and Nanqi et al. [3]. Absorption and adsorption is straightforward and economic processes [4]. Several materials have been used at adsorption processes for instance, active carbon, natural materials, and agricultural wastes [4-6]. The rapid enhancement in nanotechnology has involved them on the wastewater treatment [7-9].

Magnetic graphene nanocomposite (MGNC) was introduced for water treatment at large number literature studies as a consequence of the effective preliminary treatment to augment cleanse, and samples purify with a composite matrix [10]. Furthermore, MGNC has a considerable attention because of the fundamental scientific interest and the promising applications in magnetic fluids, catalysis, sensors, biomedicine, spintronics, magnetic recording devices, and environmental remediation [11-14]. Moreover, MGNC also shows advantages such as low toxicity, low cost, and eco-environmentally friendly. With the magnetic property, the new absorbent using magnetic solidphase extraction techniques could avoid time-consuming procedures and be performed directly to pretreat crude samples without the need for centrifugation or filtration, due to the facile magnetic separations and regeneration. From literature survey, the utilization of MGNC in water treatment at microscale devices has not been reported before.

Several methods have been developed for the synthesis of magnetic nanoparticles at different compositions such as wet chemical, template-directed, thermal decomposition co-precipitation, and solvothermal method [15-19]. Many studies have been reported the preparation of magnetic nanomaterial composites such as magnetite/SiO ${ }_{2}[20]$, magnetite/gold[21] and magnetite $/ \mathrm{SiO}_{2}[22]$. 
MGNC is one of the most reported nanocomposites because this composite matrix combines two main properties, firstly, Graphene is a 2D shape of the carbon-based material. Secondly, it shows high crystal quality and high surface area $[23,24]$.

The current study, microchannel mixer has employed for removal of dye from water. In particular, microstructure reactor or mixer, which is an essential device because its mixing has a significant effect on heat and mass transfer along with high selectivity of the reaction[25]. Generally, microfluidic technology presents many benefits, which implies substantially smaller required sample and element. As these benefits, microfluidic technology at the beginning promised extensive optimism for heat and mass transfer and biological research [21]. The field of microfluidics contracts with the performance, exact control and operation of fluids that are geometrically constrained to a small, typically sub-millimetre, scale. It is a multi-disciplinary field interconnecting manufacturing, physical science, chemistry, and nanotechnology, with applied applications to the design of arrangements in which low volumes of fluids are processed to achieve, automation, and high-throughput transmission[22]. Microfluidics developed in the beginning of the 1980s and is used in the development of inkjet printing, micro-propulsion, and micro-thermal technologies.

As several investigators have pointed out, minimizing fluidic processes to the microscale offers many significant advantages. The main benefit of microfluidic systems is the reduction in size and others a result of the capability to integrate at this scale. Another benefit of microfluidics is the low thermal mass and large surface to volume ratio of small components facilitates hasty heat transfer, allowing quick temperature changes and precise temperature control. The high sensitivity are the other advantages of microfluidic systems. Microfluidic research became one of the very attractive research areas according to its promising potentials. For the micromixers, it is anticipated to boost mixing performance with promised advantages for a decrease of the diffusion length between the reactants, growth in the area at fluid interfaces and reducing the reaction volumes. The stream in micromixers channels is often less turbulent than that in conventional large scale mixers, and the small length scales permit diffusion to play a more important role in mixing process [26]. Different channel geometries which have been introduced to improve the performance of mixing process were proposed to enhance convection mass transfer by increasing the contact area of the reactants per unit volume [27-29].

For the current investigation, presents an experimental investigation of mixing performance of various micromixers designs: T-shape, Y-shape V-shape, and KM micromixers have been completed in two parts: firstly, investigation of mixing performance of the diverse micromixers using iodideiodate reaction method or Villermaux/Dustman reaction. After studying the performance of the micromixers, the absorption of Acid blue 25 from water by using magnetic graphene has been used. MGNC has been accomplished and characterized using co-precipitation technique on Graphene Nanoplatelets. One of the Advantages of this method is that no loss of absorbent on regeneration the MGNC was utilized for wastewater treatment as the absorbent at the used microreactors. The removal capacity of MGNC was investigated at mass flow rates range from 100 to 300 ( $\mathrm{mL} / \mathrm{hr}$ ).

\section{Materials utilized}

All chemicals were purchased from Sigma-Aldrich company with high purity grades. Graphene nanoplatelets (GNPs, Strem Chemicals, Inc. USA) in addition to Iron (iii) chloride $\mathrm{FeCl}_{3}$, iron (ii) chloride tetra hydrate $99+\% \mathrm{FeCl}_{2}$, were used for preparation of $\mathrm{MCNC}$. For Villermaux/Dustman reaction experiments two solutions were prepared. Hydrochloric acid $(\mathrm{HCl})$ and Distilled Water (DW) were used for preparing the solution (1). Boric Acid $\left(\mathrm{H}_{3} \mathrm{BO}_{3}\right)$, Sodium hydroxide $(\mathrm{NaOH})$, Potassium 
lodate $\left(\mathrm{KIO}_{3}\right)$, Potassium lodide $(\mathrm{KI})$ and DW were utilized to prepare a solution (2). The information of used materials in this study are summarized in

Table 1.

Table 1

Chemical solutions that used Dushman's reaction.

\begin{tabular}{lll}
\hline component & $\begin{array}{l}\text { Concentration } \\
\text { (molar) }\end{array}$ & $\begin{array}{l}\text { Molecular Weight } \\
\text { (g / mol) }\end{array}$ \\
\hline $\mathrm{HCl}$ & 0.03 & 36.46 \\
$\mathrm{H}_{3} \mathrm{BO}_{4}$ & 0.09 & 61.83 \\
$\mathrm{NaOH}$ & 0.09 & 40.0 \\
$\mathrm{KIO}_{3}$ & 0.06 & 214.0 \\
$\mathrm{KI}$ & 0.032 & 166.0 \\
\hline
\end{tabular}

\section{Experimental methodology}

\subsection{Synthesis and characterization of the nanomaterial}

Graphene Nano-plates (GNPs) were attached with nano magnetite using co-precipitation technique with the aid of ultrasound for the production MGNC. GNPs were added to $50 \mathrm{ml}$ distilled water contains one $(\mathrm{gm})$ of iron (iii) chloride $\mathrm{FeCl} 3$ and $0.5(\mathrm{gm})$ of iron (ii) chloride tetra hydrate, $99+\% \mathrm{FeCl} 2$ ultrasonically in a bath sonicator for $30 \mathrm{~min}$ to obtain a light-brown solution. Ultrasound wave operating at $20 \mathrm{kHz}$ with a power of 500W (VCX 500, sonic materials Inc.). The solution was heated to $40{ }^{\circ} \mathrm{C}$. Then the solution of $0.2 \mathrm{M} \mathrm{NaOH}$ was added dropwise to the previously heated solution. The resulting suspension was centrifuged to separate the powder product. The black product was washed and wisely dried before proceeding to the characterization step. The process of MGNCs fabrication is revealed at Fig. 1 . The fabricated composite has been characterized by X-ray diffraction (XRD-6100 Shimadzu, Japan) equipped with $\mathrm{Cu} K \alpha$ radiation source with wavelength $(\lambda=$ $1.5418 \AA$ A). The morphology of produced composite has been estimated by Transmission Electron Microscope (TEM) (TEM, JEM-2100). The magnetic properties were analysed using Vibrating Sample Magnetometer (VSM) at room temperature. The additional functional information of graphene sample was measured by Fourier Transform Infrared/IR spectrometer (FTIR) (Vertex 70, Bruker scientific instruments, Germany). The specific surface areas were measured using Brunauer-EmmettTeller (BET) with an ASAP 2020 Micrometrics Instrument at $77 \mathrm{~K}$. 


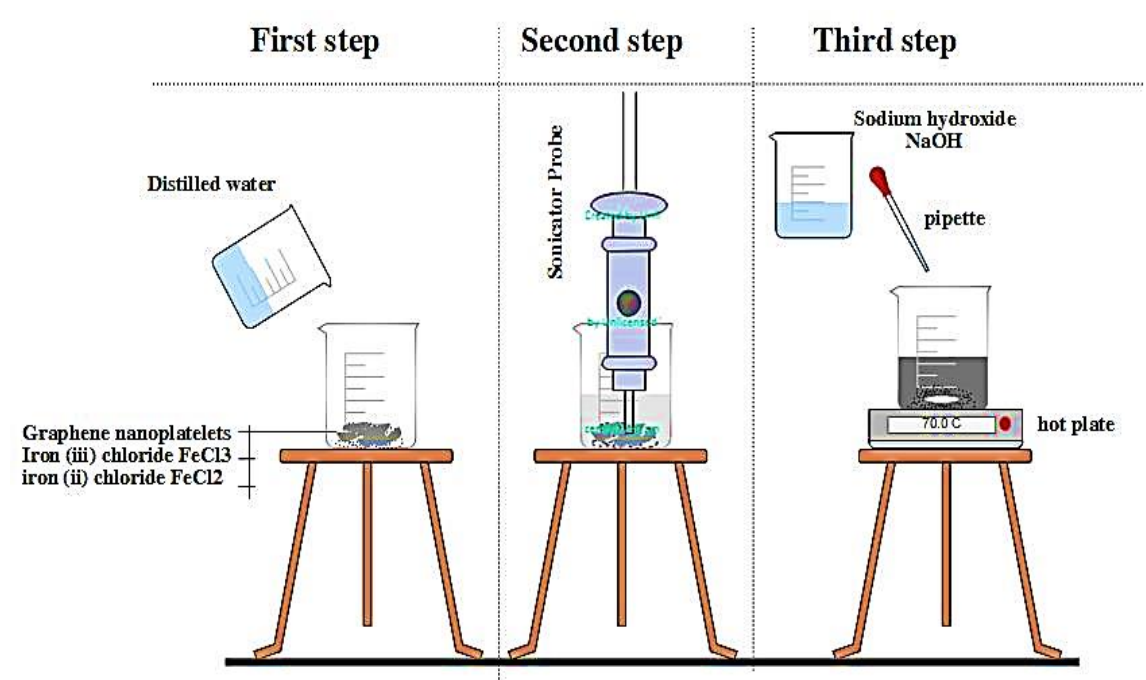

Fig. 1. Preparation of graphene magnetite Composite steps

\subsection{Evaluation of mixing performance}

The experimental setup for measuring mixing performance of various micromixers is shown in Fig. 2 . Firstly, the syringe (KDS 100 LEGACY KD, Scientific Inc. U.S.A) was utilized in which the reaction flow rate was controlled, and the two syringes were filled with the two solutions. The connectors were used to assembly microreactor with pipes and syringes. All the micromixers were supplied from Kyoto University. T-shape, Y-shape, and V-shape which were fabricated by 3D-printer technology

Table 2.

The required flow rate was adjusted at the pump, in addition to the dimensions of syringes. The two solutions were pumped at the micromixer and wait while the steady state accomplished then the samples were collected. UV/Visible spectrophotometer (Acculab UVS-90 Wavelength range 190 1100 $\mathrm{nm}$ Spectral bandwidth $2 \mathrm{~nm}$ ) was operated to measure the adsorption of the collected samples at a wavelength of $352 \mathrm{~nm}$.

The mixing time is subject to reaction conditions, and it only can be assessed for each experiment using micro mixing models. Several authors have established an "integration model" to calculate $t_{m}$ from the segregation index, in many kinds of reactors. According to jean-marc and Laurent Falk study [31], they organized their work and recommended a step-by-step methodology to apply villermauxDushman's protocol at micromixers to estimate the mixing time.

Mixing times or experimental mixing time was obtained using the following equations Eq. 1 where (OD) is the optical density that was obtained from UV spectrometer at wavelength $\lambda=352 \mathrm{~nm}$. The optical density is a function of sample thickness (L) as indicated from Eq. 2

$$
t_{m}=0.33(\mathrm{OD})\left[\mathrm{H}^{+}\right]^{-.4 .55}[\mathrm{KI}]^{-1.5}\left[\mathrm{KIO}_{3}\right]^{5.8}[\mathrm{NaOH}]^{-2}\left[\mathrm{H}_{3} \mathrm{BO}_{3}\right]^{-2}
$$

$O D=A b s / L$

where, (Abs) is the absorbance measurement of the samples.

The same test setup was used to study the adsorption characteristics of the MGNC for removal of the acid Blue 25 dye. The polluted water was filled in one syringe, and the other syringe included the MGNC nanofluid. The two fluids were pumped to the micromixers, and the samples were collected and instantaneously centrifuged, and the absorption of the dye was measured Using UV spectrometer at $\lambda=600 \mathrm{~nm}$. The percentage removal of dye was calculated using the Eq. 3 . Where $\%$ 
$\mathrm{R}$ is the percentage of dye adsorption, $\mathrm{C}_{0}$ is the initial dye concentration, and $\mathrm{C}$ is the final dye concentration after contact with the MGNC.

$\% \mathrm{R}=\frac{C_{0}-C}{C_{0}} \times 100$
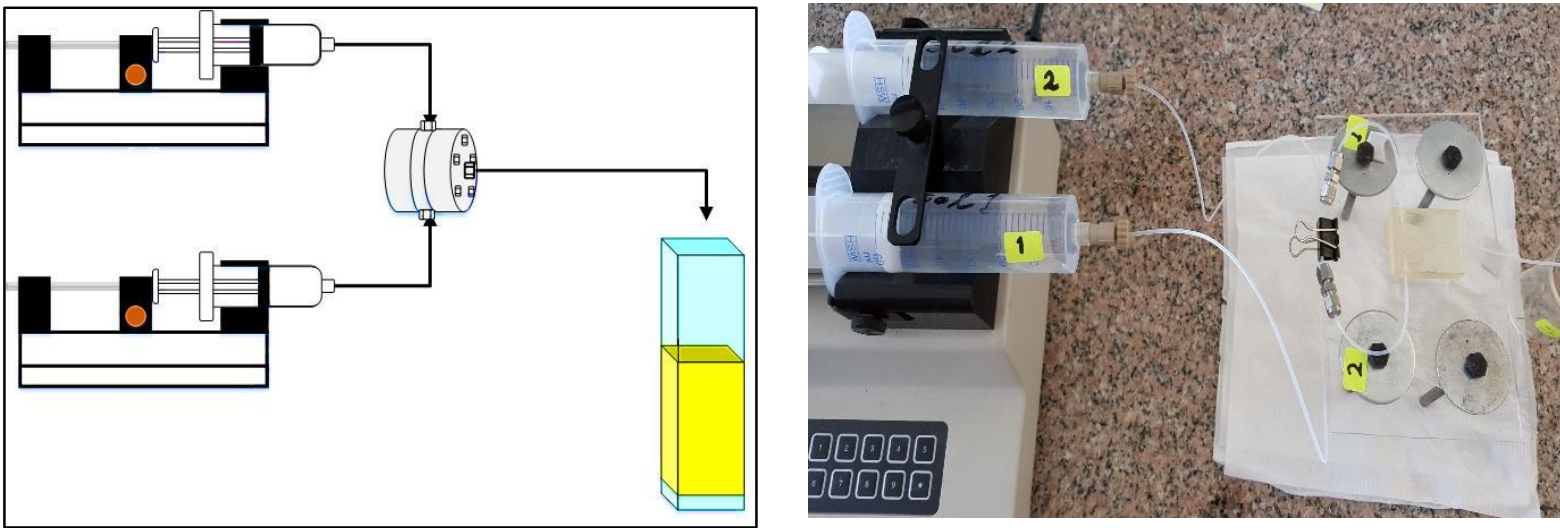

Fig. 2. The schematic and actual experimental setup for measuring mixing performance

Table 2

Details of Micromixers

\begin{tabular}{|l|l|l|l|l|}
\hline Mixer Type & Microscope Photo & Actual Photo & $\begin{array}{l}\text { Average dimension } \\
\text { of channels }(\mu \mathrm{m})\end{array}$ \\
\hline 1) T-mixer & & & & 464 \\
\hline \multirow{2}{*}{ 2) Y-mixer } & & & & \\
\hline
\end{tabular}




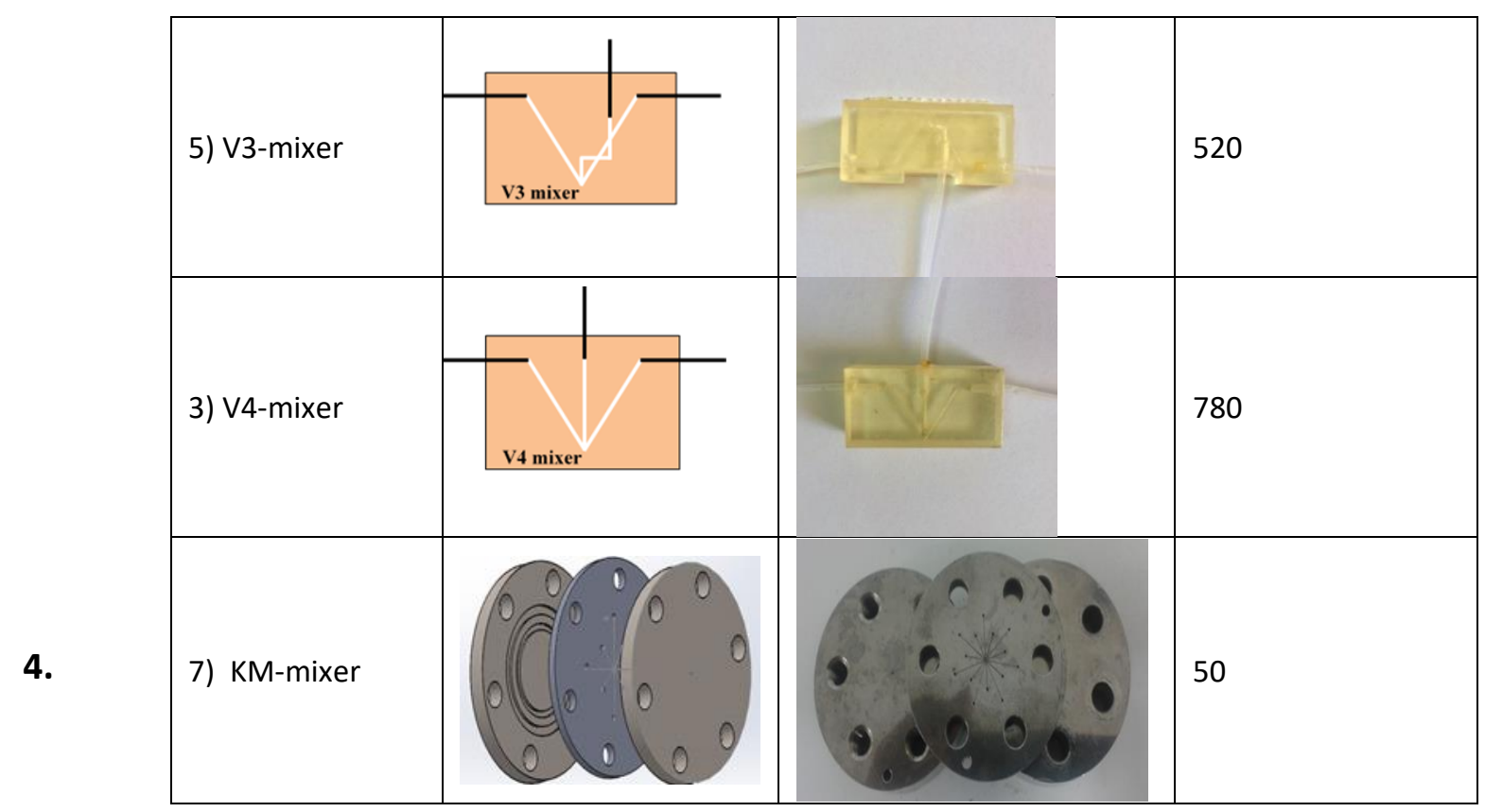

\section{Results and discussion}

\subsection{Preparation and characterization of MGNC.}

The different characterization techniques showed that Graphene Nanoplatelets (GNPs) was immobilized with Nano-magnetite using co-precipitation technique with the aid of ultrasound. The various physical and chemical properties of fabricated magnetic graphene composite were revealed. The physio-chemical characterization confirmed the successful preparation of MGNC by illustration the crystalline and chemical structures of Nano graphene after magnetic immobilization. Moreover, the superparamagnetic properties of the prepared composite were confirmed by the measurements of magnetic properties.

The crystalline structures of MGNC matrices are illustrated by XRD which are illustrated at

Fig. 3. It was concluded that the XRD pattern of as-synthesized MGNCs shows that all the characteristic diffraction peaks which are in good agreement with those of $\mathrm{Fe}_{3} \mathrm{O}_{4}$ (Magnetite, PDF, $00-026-1136)[15,33]$, in addition to the peak of graphene that appears at $2 \theta=26.223^{\circ}[34]$.

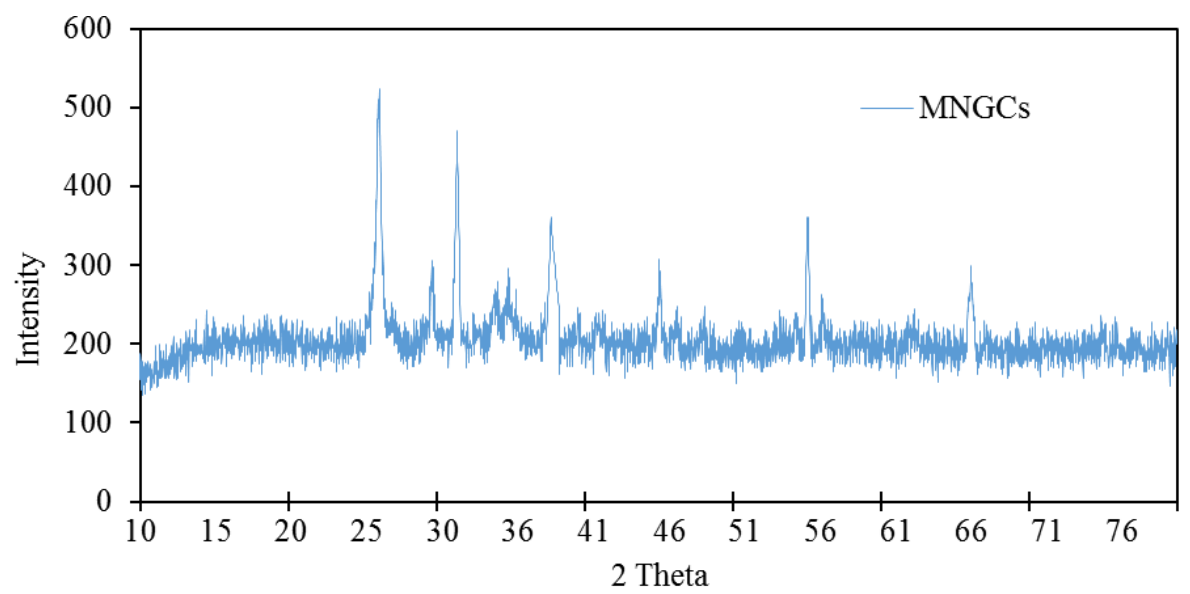

Fig. 3. XRD pattern of prepared MGNCs Nano composites 
Fig. 4 shows FTIR spectra of MGNC characteristics and shows new absorption bands at $463 \mathrm{~cm}^{-1}$ that represents ( $C-C=O$ in-plane deformation vibration) and another peak around $600 \mathrm{~cm}-1$ results from the split of the $u 1$ band at $598 \mathrm{~cm}^{-1}$, which corresponds to the Fe-O bond of bulk magnetite. These results confirm that $\mathrm{Fe}_{3} \mathrm{O}_{4}$ has been successfully prepared on the surface of graphene[35]. The peak located at $1708.93 \mathrm{~cm}-1$ has been regarded to stretch the $\mathrm{C}=0$ stretching [36,37]. Also peaks at 3732,3628 and $3410 \mathrm{~cm}^{-1}$ represent (O-H stretching) which confirm the presence of the functional groups containing oxygen in carbon frameworks. These results are submitting the evidence of the successful synthesis of immobilising of $\mathrm{Fe}_{3} \mathrm{O}_{4}$ at Graphene surface.

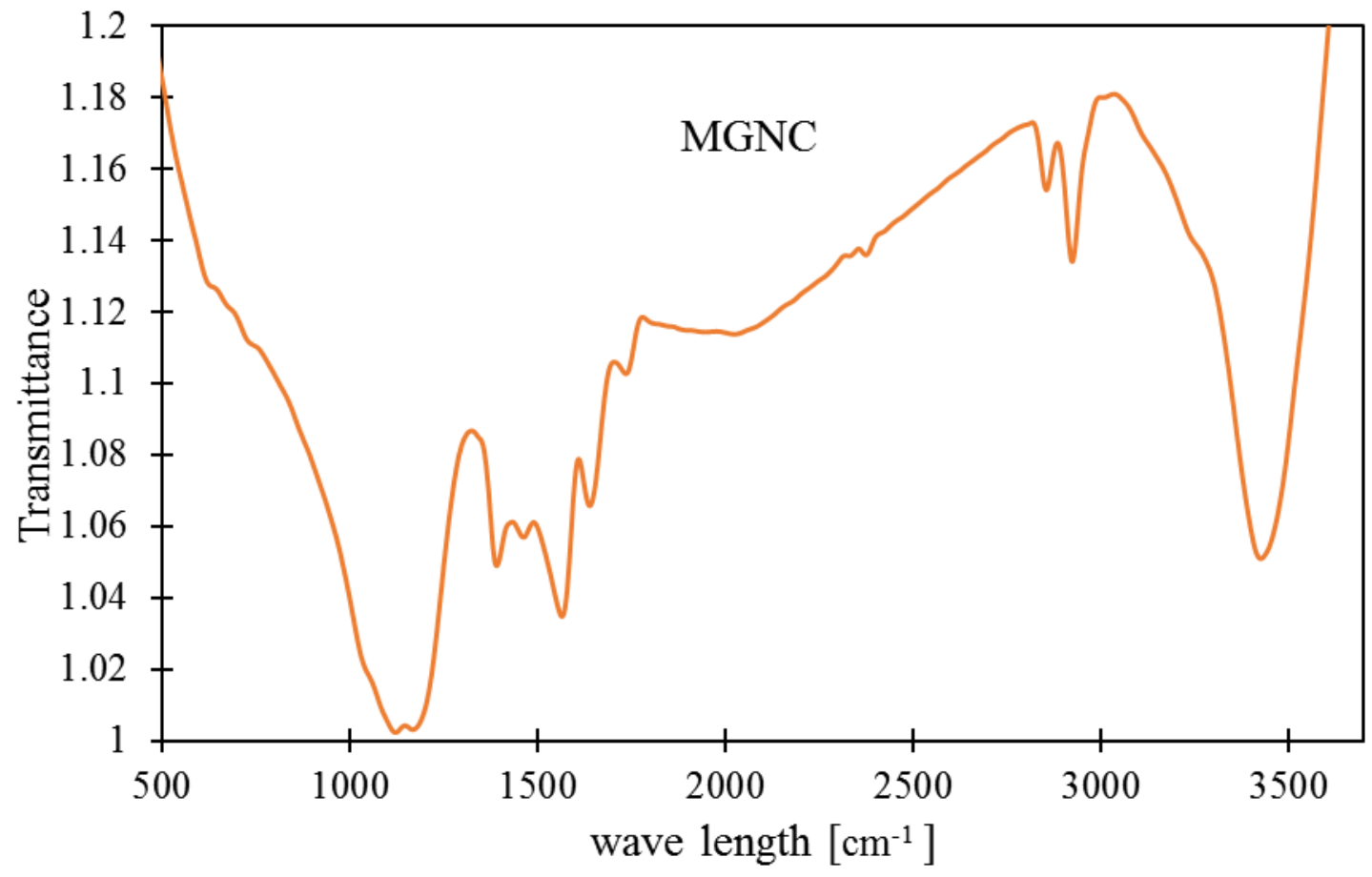

Fig. 4. FTIR spectra of MGNCs.

The TEM imaging characterized the morphologies of the GNPs and MGNCs. Fig. 5a illustrate the TEM image of pure graphene which shows the nanoplatelets surfaces such as a soft wrinkled surface [38]. Moreover, GNP showed a flake structure with several in-plane sizes and specified regular graphene layers. The TEM observation revealed the 2D shape of graphene which is a carbon based material existing as a monolayer arranged in a honeycomb lattice [39], and the thickness of GNP is around $10 \mathrm{~nm}$. The high degree of exposed surface at active sides of graphene to be an excellent template at co-precipitation method which was reveal by the TEM. The images of MGNCs, as shown in Fig. 5b, Fig. $5 \mathrm{c}$ and Fig. $5 \mathrm{~d}$, reveal that the synthesized magnetite $\left(\mathrm{Fe}_{3} \mathrm{O}_{4}\right)$ nanoparticles formation. Accordingly, these images confirm their uniformly distribution on the plane of a graphene platelets, and the magnetite particles successfully have wrapped around the surfaces of graphene. 

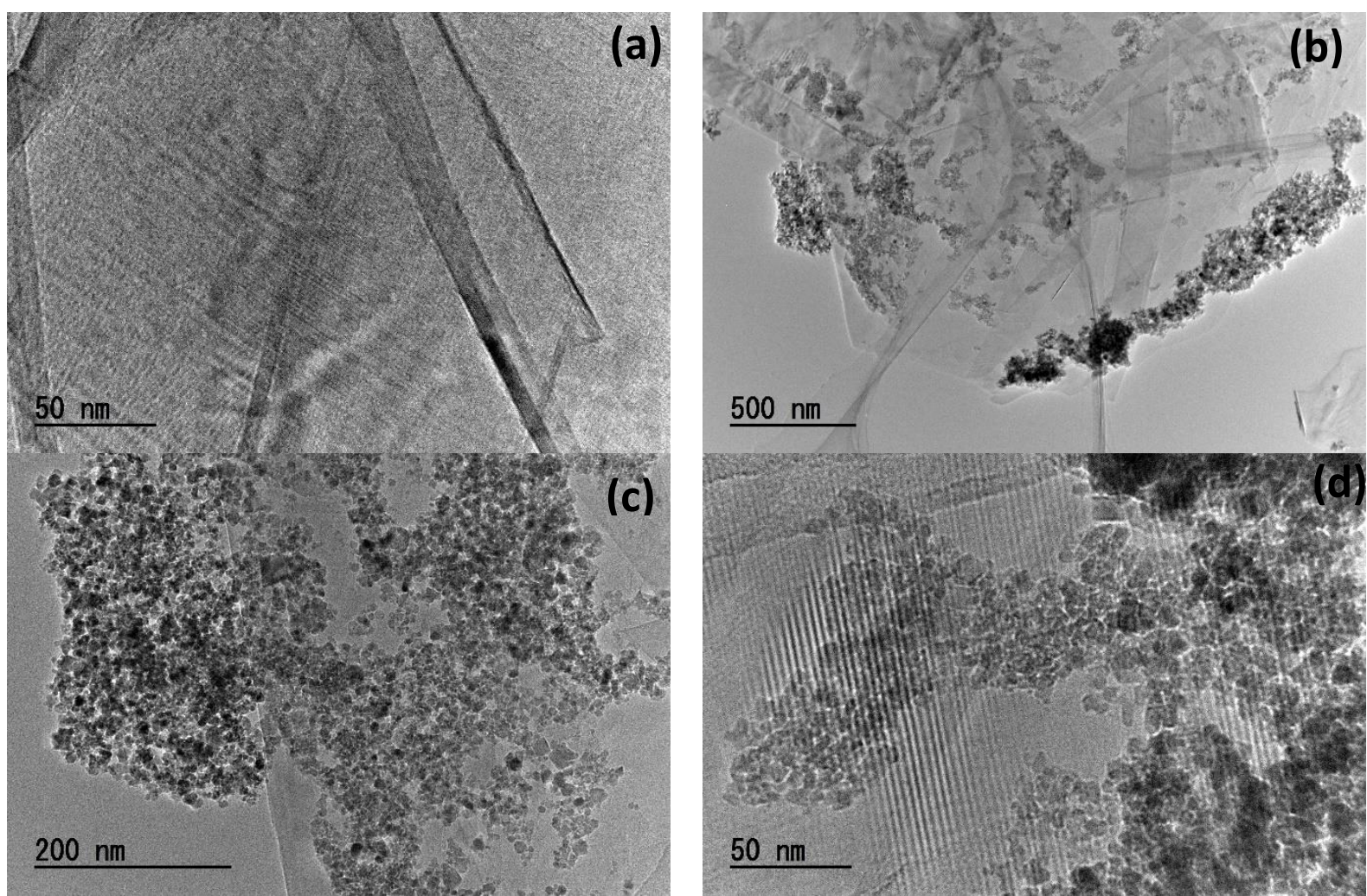

Fig. 5. TEM images of (a) GNPs and (b, $c$, and c) MGNCs at different magnifications

BET analyses have been used to measure the specific surface areas for GNPs and MGNCs, using Barrett-Joyner-Helena (BJH) method. Table 3) shows BET values that indicated the surface area and the total pore volume of MNGCs were larger than that of the GNPs material. On the other hand, BET values of MGNCs show the increase of surface area up to $34.389 \mathrm{~m}^{2} / \mathrm{g}$ and the mean pore diameter up to $14.949 \mathrm{~nm}$ of the magnetite immobilisation compared with GNPs.

\section{Table 3}

Nitrogen adsorption and desorption analyses of GNPs and MGNCs.

\begin{tabular}{llllll}
\hline Morphology & BET Surface Area $\left(\mathrm{m}^{2} / \mathrm{g}\right)$ & $\begin{array}{l}\text { Total Pore Volume*10- } \\
3\left(\mathrm{~cm}^{3} / \mathrm{g}\right)\end{array}$ & $\begin{array}{l}\text { Average } \\
(\mathrm{nm})\end{array}$ & Pore Size \\
\hline GNPs & 15.965 & 7.5115 & 18.82 & \\
\hline MGNCs & 34.389 & 13.04 & 14.949 & \\
\hline
\end{tabular}

The magnetic properties of the MGNCs were investigated using VSM at room temperature (25 C). The hysteresis loop of the synthesized MGNCs were recorded and observed. As shown in Fig. 6 the MGNCs material shows typical superparamagnetic properties. The saturation moment per unit mass, (Ms) for the MGNCs is $22.7548 \mathrm{emu} \cdot \mathrm{g}^{-1}[40]$. 


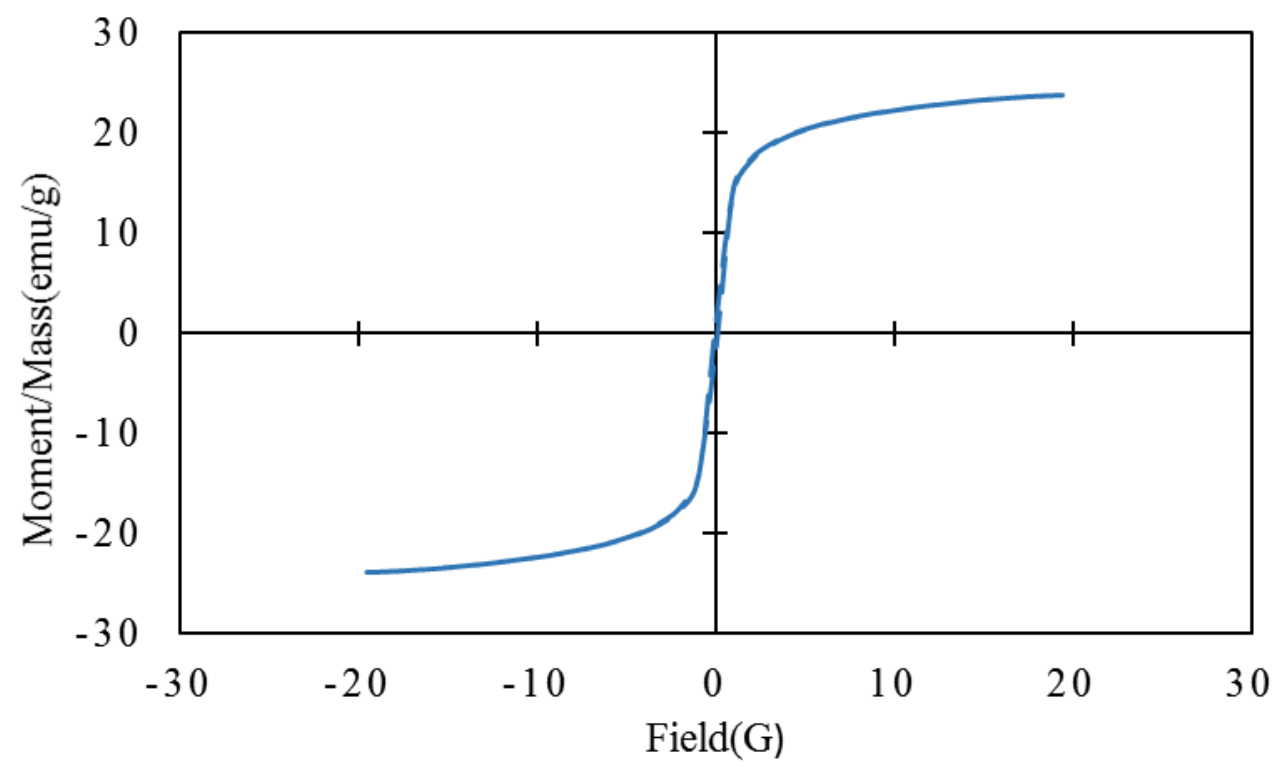

\subsection{Mixing Performance}

Fig. 6. Magnetization curves of MGNCs

There are main types of mixing junction $T, Y$ and $V$ were tested at $T$ junction the two inlets are in the same line and the $V$ junction inlets flow coming in be angle this making a turbulence at mixing area and the mixing area become bigger than that at $T$ Junction. In fact, $Y$ and $V$ Junction are the same at inlets but they are different at their outlet. The $V$ shape characterized by its turbulence at mixing area is larger than that of $Y$ shape. Hence, comparing these micromixers with KM-mixer, its inlet is multi-channel inlets which are $\mathrm{V}$ shaped so it represents the better mixing performance.

In order to estimate the influence of the volumetric flow rate and geometric design on the performance mixing, the values of absorbance for T-mixers were compared with Y-mixers, and K-M mixer. According to Fig. 10, the absorbance values of the various micromixers at different flow rates is obvious that confluence angle $(\theta)$ has a significant influence on the mixing performance. It is obvious that, the absorbance intensity frequently decreased with increasing the flow rate at all mixer except K-M mixer the influnce of flow rate change it can be neglected. From Error! Reference source not found., T-mixer shows the highest absorbance values which means that the mixing was imperfect even at the highest flow rate $300 \mathrm{ml} / \mathrm{hr}$. Fig. 7 shows that the Y-mixer has the same trend of absorbance change with flow rate change. According to absorbance measurement, Y-mixer revealed a better mixer compared with the T-mixer. On the other hand, V-shaped mixers mixing performance was better than $T$ and $Y$ designs, and also the mixing performance was enhanced by increasing the flow rate at $300 \mathrm{ml} / \mathrm{hr}$ the mixing performance is reasonable at the absorbance values were low, which means the little amount of $I_{3}^{-}$was formed from the second reaction. In contrast, K-M micromixer from, 
Fig. 9 exhibited outstanding mixing performance at all flow rate range even at low flow rate values these results match with Nagasawa et al.[25]

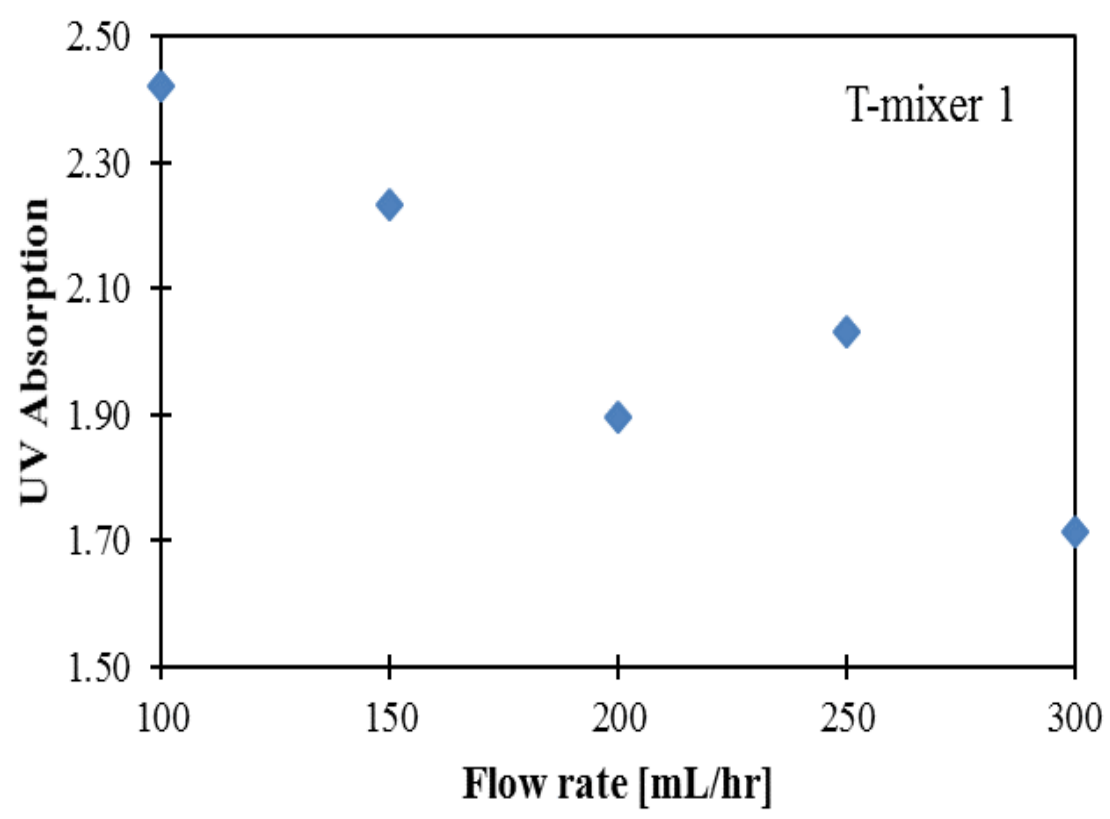

Fig. 7. Change of absorption of the mixing with the flow rate at T-mixer.

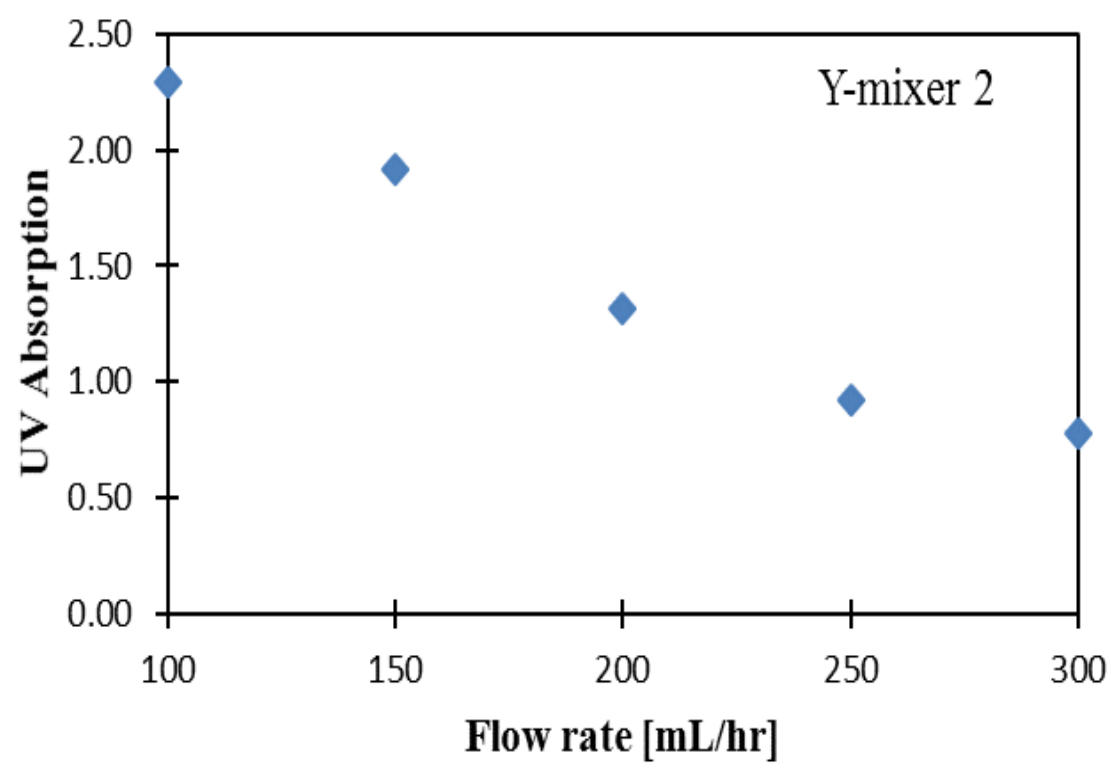

Fig. 7. Change of absorption of the mixing with the flow rate at $\mathrm{Y}$-mixer. 


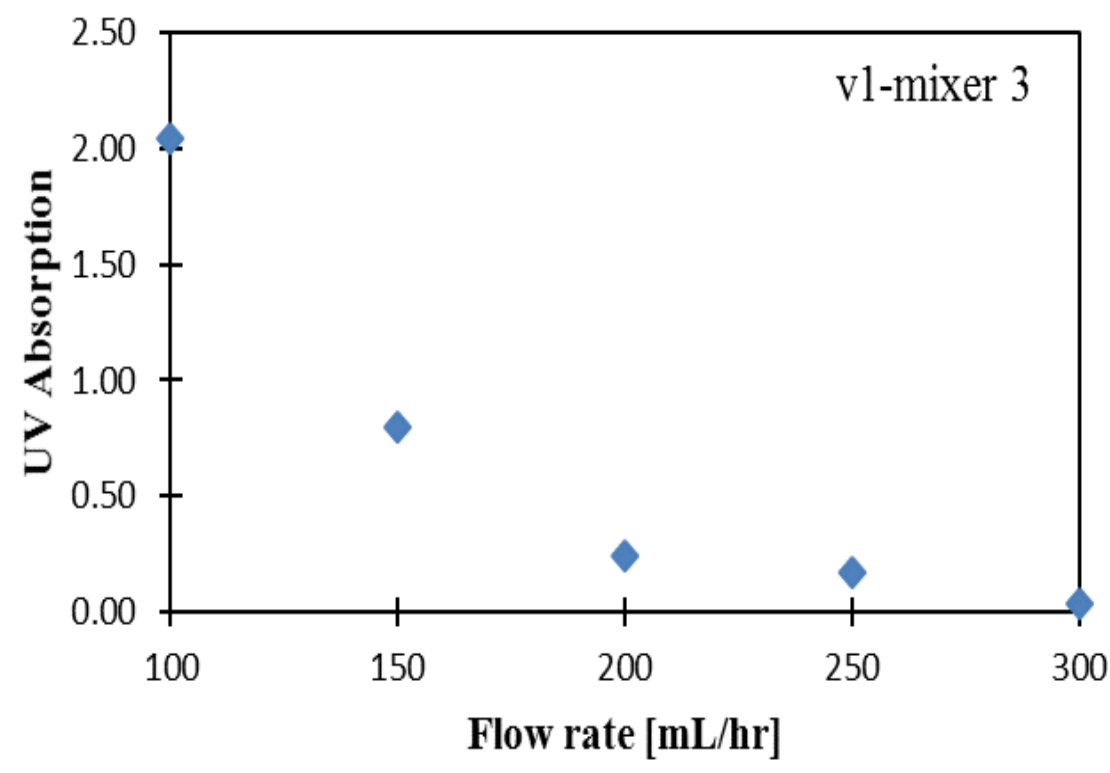

Fig. 8. Change of absorption of the mixing with the flow rate at v1-mixer.

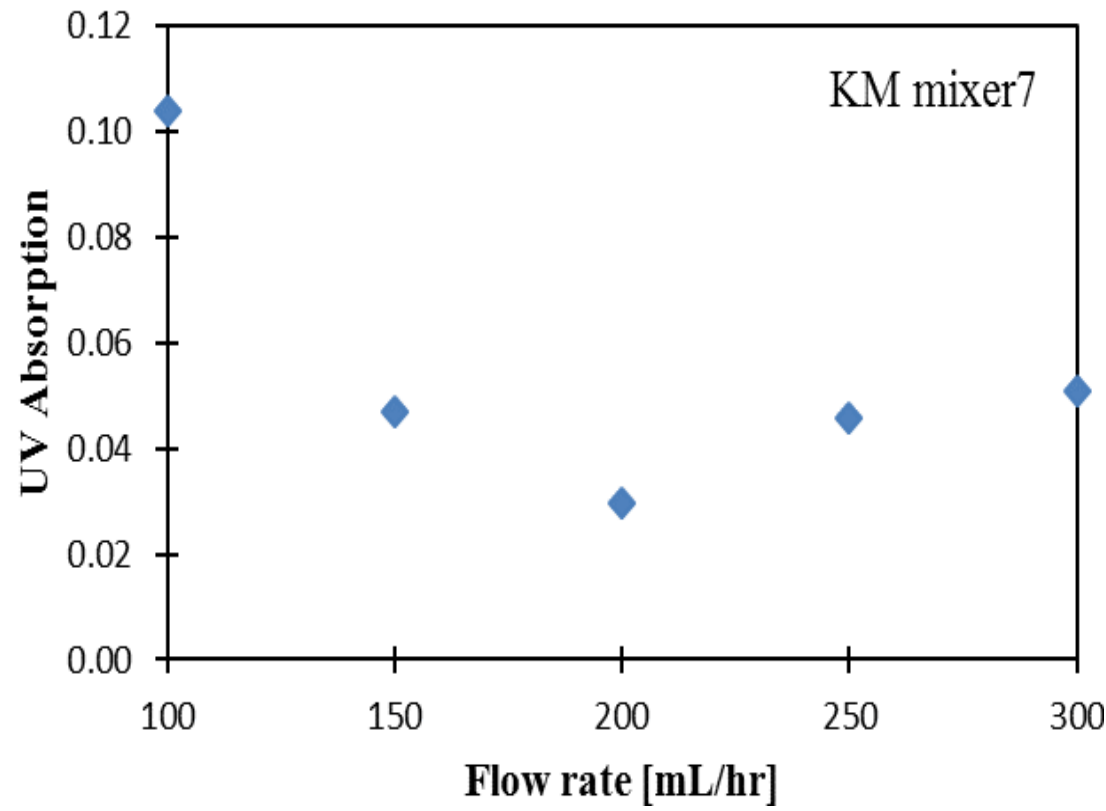

Fig. 9. Change of absorption of the mixing with the flow rate at K-M mixer 
$\diamond \mathrm{KM} \square \mathrm{T} \Delta \mathrm{Y} X \mathrm{~V} 1 * \mathrm{~V} 2 \bullet \mathrm{V} 3+\mathrm{V} 4$

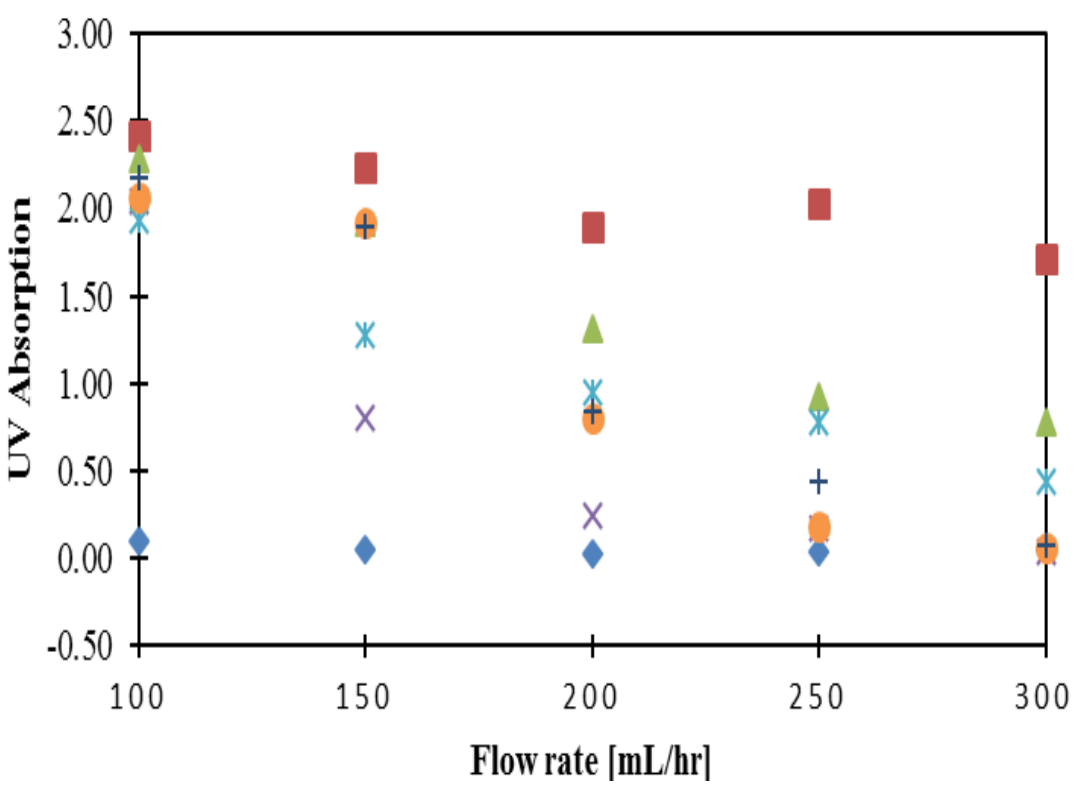

Fig. 10. Change of absorption of the mixing with the flow rate

\subsection{Mixing time determination}

To confirm a well understanding of the micro mixing effectiveness in microreactors, mixing time $\left(t_{m}\right)$, was considered as a valuable parameter for evaluating the mixing. In other words, long mixing time means that the mixing is imperfect and vice versa.

Fig. 11 demonstrates that mixing time was effected by changing the volumetric flow rate. When the flow rate increased, the mixing time decreased at all mixers types. At the T mixer, the mixing time at all flow rates was long, for instance, the higher value of tm was $0.47 \mathrm{sec}$ at $100 \mathrm{ml} / \mathrm{hr}$ and it was $0.17 \mathrm{sec} 300 \mathrm{ml} / \mathrm{hr}$. The K-M mixer exposed very short mixing time which approves that this mixer has an identical upright performance. It is obvious that changing the volumetric flow rate at K-M mixer has a little influence at mixing time because as shown in Fig. 11.

$\diamond \mathrm{KM} \square \mathrm{T} \triangle \mathrm{Y} \times \mathrm{V} 1 * \mathrm{~V} 2 \bullet \mathrm{V} 3+\mathrm{V} 4$

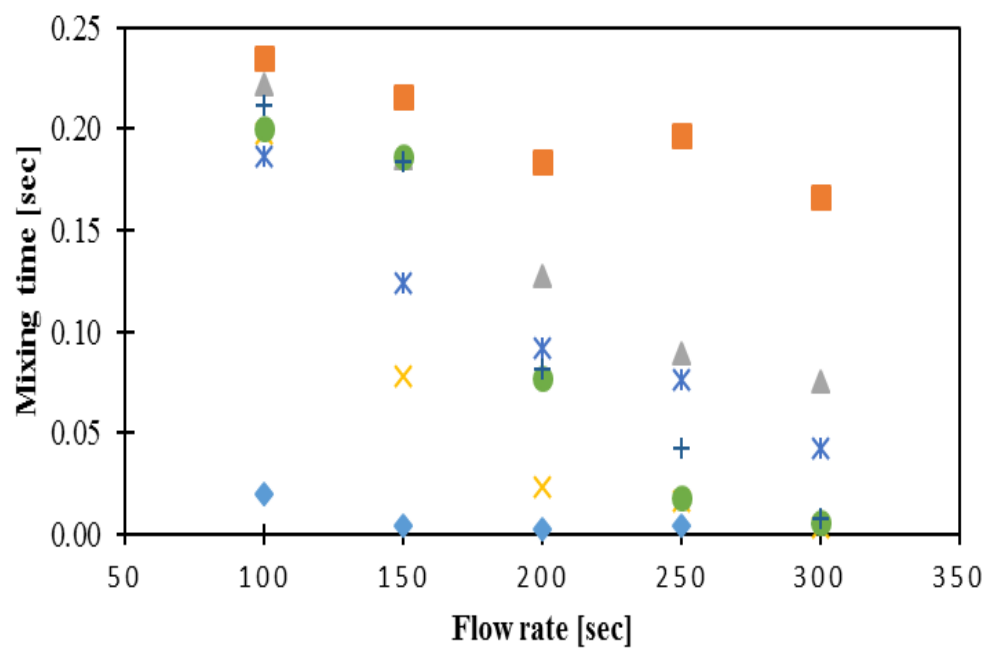

Fig. 11. Mixing time change with flow rate of all mixer. 


\subsection{Water treatment}

Fig. 12 compares real samples of pure water, initial water with dye and treated water. It is clear to observe the effect of using MGNC at dye removal. As shown in Fig. 13, the highest dye removal values have been achieved with K-M Mixer, and the lowest values of removal were at T-Mixer, which exhibited low ability to remove the dye from the water. These results are strongly agreeing with what expected by Dushman's reaction experiments. On the other hand, MGNC is an efficient adsorbent for pollutants removal it shows a satisfactory performance by using it micromixers the removal percentage quite small according to the short mixing time, and the adsorption process is a slow one.

The optimal instantaneous removal capacity of graphene using K.M micromixer was about $68 \%$ for $10 \mathrm{ppm}$ dye concentration. The T-mixer showed low removal values according to its defective mixing, the highest value of removal at T-mixer was around $22 \%$.

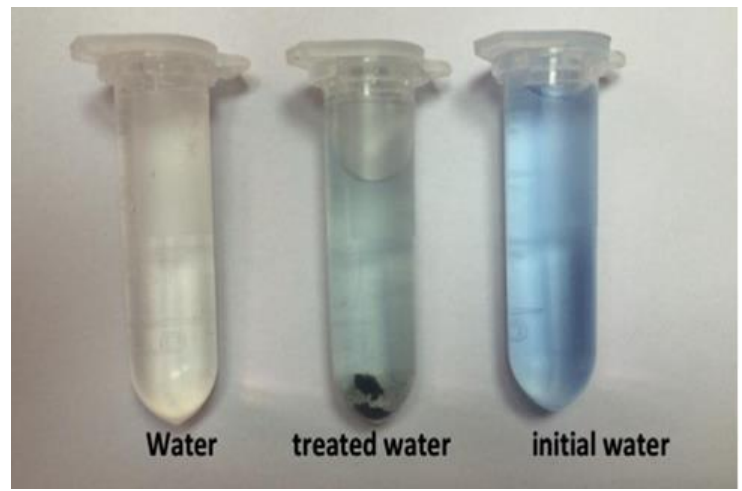

Fig. 12. Comparison between pure water, initial water with dye and treated water.

$\square \mathrm{T} \triangle \mathrm{Y} \times \mathrm{V} 1 * \mathrm{KM}$

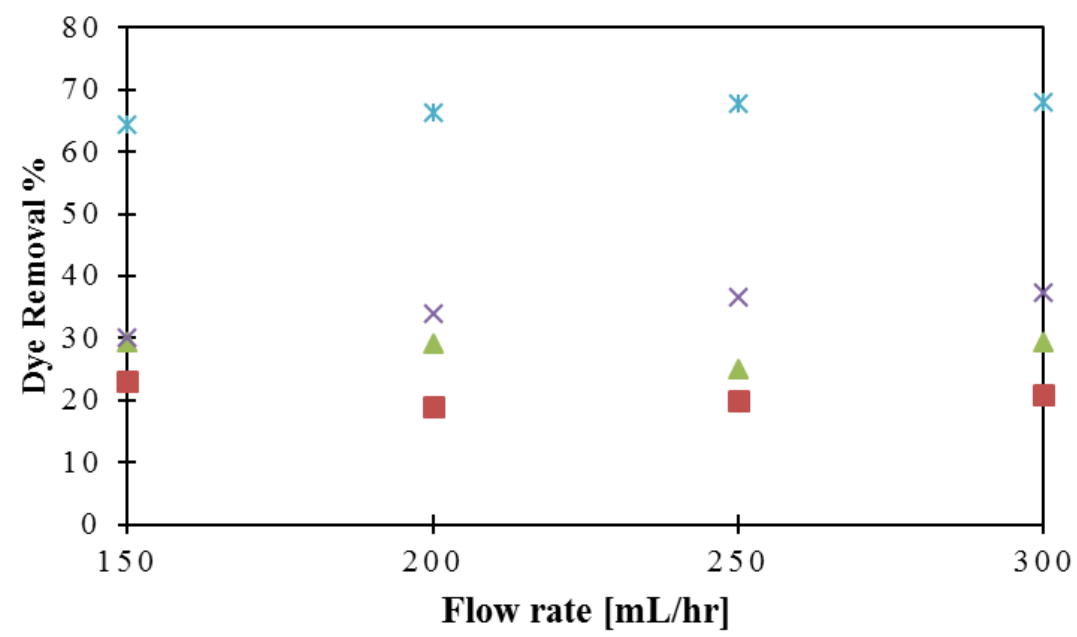

Fig. 13. Change of dye removal the flow rate.

\section{Conclusion}

A magnetic graphene nanocomposite MGNCs has been successfully produced by co-precipitation technique. The mixing performance of the seven various design microreactors using the Dushman's method was studied-M mixer has the perfect mixing performance compared with the others used mixer types. The mixing time for the micromixers was determined at flow rates ranged from 100 to $300 \mathrm{ml} / \mathrm{hr}$. The graphene-based nanomaterials which were employed to remove an Acid Blue 25 dye from polluted wastewater. The highest removal capacity of graphene using was obtained by K.M 
micromixer which was around $68 \%$ at $300 \mathrm{ml} / \mathrm{hr}$ flow rate. The T-mixer showed low removal values, for example, it was about $22 \%$ at $300 \mathrm{ml} / \mathrm{hr}$.

\section{References}

[1] Srinivasan, Asha, and Thiruvenkatachari Viraraghavan. "Decolorization of dye wastewaters by biosorbents: a review." Journal of environmental management 91, no. 10 (2010): 1915-1929. https://doi.org/10.1016/j.jenvman.2010.05.003

[2] Mondal, S. "Methods of dye removal from dye house effluent-an overview." Environmental Engineering Science 25, no. 3 (2008): 383-396. https://doi.org/10.1089/ees.2007.0049

[3] Ren, Nanqi, Xianjiao Zhou, Wanqian Guo, and Shanshan Yang. "A review on treatment methods of dye wastewater [J]." CIESC Journal 1 (2013). https://doi.org/10.3969/j.issn.0438-1157.2013.01.011

[4] Bitton, G. "Introduction to wastewater treatment en Wastewater microbiology." (2005): 213-223. https://doi.org/10.1002/0471717967.ch7

[5] Kaegi, Ralf, Andreas Voegelin, Brian Sinnet, Steffen Zuleeg, Harald Hagendorfer, Michael Burkhardt, and Hansruedi Siegrist. "Behavior of metallic silver nanoparticles in a pilot wastewater treatment plant." Environmental science \& technology 45, no. 9 (2011): 3902-3908. https://doi.org/10.1021/es1041892

[6] Bauer, Christian, Thomas Heck, Niels Jungbluth, and Thomas Nemecek. "The environmental relevance of capital goods in life cycle assessments of products and services." (2007). https://doi.org/10.1016/S0026-0576(09)80216-X

[7] Mahmoud, Mohamed E., Mohamed S. Abdelwahab, and Eiman M. Fathallah. "Design of novel nano-sorbents based on nano-magnetic iron oxide-bound-nano-silicon oxide-immobilized-triethylenetetramine for implementation in water treatment of heavy metals." Chemical Engineering Journal 223 (2013): 318-327. https://doi.org/10.1016/j.cej.2013.02.097

[8] Tesh, Sarah J., and Thomas B. Scott. "Nano-composites for water remediation: A review." Advanced Materials 26, no. 35 (2014): 6056-6068. https://doi.org/10.1002/adma.201401376.

[9] Terzi, Evren, S. Nami Kartal, Nural Yılgör, Lauri Rautkari, and Tsuyoshi Yoshimura. "Role of various nano-particles in prevention of fungal decay, mold growth and termite attack in wood, and their effect on weathering properties and water repellency." International Biodeterioration \& Biodegradation 107 (2016): 77-87. https://doi.org/10.1016/j.ibiod.2015.11.010

[10] Upadhyay, Ravi Kant, Navneet Soin, and Susanta Sinha Roy. "Role of graphene/metal oxide composites as photocatalysts, adsorbents and disinfectants in water treatment: a review." RSC advances 4, no. 8 (2014): 38233851. https://doi.org/10.1039/c3ra45013a.

[11] Abo-Zahhad, E. M., Ahmed H. El-Shazly, and M. F. El-Kady. "Synthesis and Characterization of Nanomagnetic Graphene via Co-Precipitation Technique with Aid of Ultrasound." In Materials Science Forum, vol. 860, pp. 21-24. Trans Tech Publications Ltd, 2016. https://doi.org/10.4028/www.scientific.net/MSF.860.21

[12] Bahiraei, Mehdi, and Morteza Hangi. "Flow and heat transfer characteristics of magnetic nanofluids: a review." Journal of Magnetism and Magnetic Materials 374 (2015): 125-138. https://doi.org/10.1016/j.jmmm.2014.08.004

[13] Dong, Yucheng, Kam Chuen Yung, Ruguang Ma, Xia Yang, Ying-San Chui, Jong-Min Lee, and Juan Antonio Zapien "Graphene/acid assisted facile synthesis of structure-tuned Fe3O4 and graphene composites as anode materials for lithium ion batteries." Carbon 86 (2015): 310-317. https://doi.org/10.1016/j.carbon.2015.01.062

[14] Jin, Bo, Guangyi Chen, Xiaobin Zhong, Yang Liu, Kaiyuan Zhou, Peng Sun, Peng Lu, Wanxi Zhang, and Jicai Liang. "Graphene/Fe3O4 hollow sphere nanocomposites as superior anode material for lithium ion batteries." Ceramics International 40, no. 7 (2014): 10359-10365. https://doi.org/10.1016/j.ceramint.2014.03.009

[15] Zhou, Guangmin, Da-Wei Wang, Feng Li, Lili Zhang, Na Li, Zhong-Shuai Wu, Lei Wen, Gao Qing Lu, and Hui-Ming Cheng. "Graphene-wrapped Fe3O4 anode material with improved reversible capacity and cyclic stability for lithium ion batteries." Chemistry of Materials 22, no. 18 (2010): 5306-5313.

https://doi.org/10.1021/cm101532x 
[16] Wang, Lei, Ying Huang, Xu Sun, Haijian Huang, Panbo Liu, Meng Zong, and Yan Wang. "Synthesis and microwave absorption enhancement of graphene@Fe 30 4@SiO 2@ NiO nanosheet hierarchical structures." Nanoscale 6, no. 6 (2014): 3157-3164.

https://doi.org/10.1039/c3nr05313j

[17] Hu, Chuangang, Zhongyu Mou, Gewu Lu, Nan Chen, Zelin Dong, Minjia Hu, and Liangti Qu. "3D graphene-Fe 304 nanocomposites with high-performance microwave absorption." Physical Chemistry Chemical Physics 15, no. 31 (2013): 13038-13043.

https://doi.org/10.1039/c3cp51253c

[18] Zhu, Shenmin, Jingjing Guo, Junping Dong, Zhaowen Cui, Tao Lu, Chenglin Zhu, Di Zhang, and Jun Ma. "Sonochemical fabrication of Fe304 nanoparticles on reduced graphene oxide for biosensors." Ultrasonics sonochemistry 20, no. 3 (2013): 872-880. https://doi.org/10.1016/j.ultsonch.2012.12.001

[19] Teo, P. S., H. N. Lim, N. M. Huang, C. H. Chia, and I. Harrison. "Room temperature in situ chemical synthesis of Fe304/graphene." Ceramics International 38, no. 8 (2012): 6411-6416. https://doi.org/10.1016/j.ceramint.2012.05.014

[20] Wang, Jiahong, Shourong Zheng, Yun Shao, Jingliang Liu, Zhaoyi Xu, and Dongqiang Zhu. "Amino-functionalized Fe304@ SiO2 core-shell magnetic nanomaterial as a novel adsorbent for aqueous heavy metals removal." Journal of colloid and interface science 349, no. 1 (2010): 293-299.

https://doi.org/10.1016/j.jcis.2010.05.010

[21] Liang, Wenbin, Weijing Yi, Yan Li, Zhujun Zhang, Mingzhen Yang, Chuanmin Hu, and An Chen. "A novel magnetic Fe304@ gold composite nanomaterial: Synthesis and application in regeneration-free immunosensor." Materials Letters 64, no. 23 (2010): 2616-2619.

https://doi.org/10.1016/j.matlet.2010.08.013

[22] Hui, Chao, Chengmin Shen, Jifa Tian, Lihong Bao, Hao Ding, Chen Li, Yuan Tian, Xuezhao Shi, and Hong-Jun Gao. "Core-shell Fe 304 S SiO 2 nanoparticles synthesized with well-dispersed hydrophilic Fe 304 seeds." Nanoscale 3, no. 2 (2011): 701-705. https://doi.org/10.1039/c0nr00497a

[23] Liu, Chenguang, Zhenning Yu, David Neff, Aruna Zhamu, and Bor Z. Jang. "Graphene-based supercapacitor with an ultrahigh energy density." Nano letters 10, no. 12 (2010): 4863-4868. https://doi.org/10.1021/nl102661q

[24] Li, Xuesong, Weiwei Cai, Jinho An, Seyoung Kim, Junghyo Nah, Dongxing Yang, Richard Piner et al. "Large-area synthesis of high-quality and uniform graphene films on copper foils." science 324, no. 5932 (2009): 1312-1314. https://doi.org/10.1126/science.1171245

[25] Nagasawa, Hideharu, Nobuaki Aoki, and Kazuhiro Mae. "Design of a new micromixer for instant mixing based on the collision of micro segments." Chemical Engineering \& Technology: Industrial Chemistry-Plant EquipmentProcess Engineering-Biotechnology 28, no. 3 (2005): 324-330. https://doi.org/10.1002/ceat.200407118

[26] Martínez-López, J. Israel, Mauricio Mojica, Ciro A. Rodríguez, and Héctor R. Siller. "Xurography as a rapid fabrication alternative for point-of-care devices: Assessment of passive micromixers." Sensors 16, no. 5 (2016): 705. https://doi.org/10.3390/s16050705

[27] Fan, Liang-Liang, Xiao-Liang Zhu, Hong Zhao, Jiang Zhe, and Liang Zhao. "Rapid microfluidic mixer utilizing sharp corner structures." Microfluidics and Nanofluidics 21, no. 3 (2017): 36. https;//doi.org/10.1007/s10404-017-1874-y

[28] Fan, Liang-Liang, Xiao-Liang Zhu, Hong Zhao, Jiang Zhe, and Liang Zhao. "Rapid microfluidic mixer utilizing sharp corner structures." Microfluidics and Nanofluidics 21, no. 3 (2017): 36. https://doi.org/10.1063/1.4870253

[29] Lin, Che-Hsin, Chien-Hsiung Tsai, Chih-Wen Pan, and Lung-Ming Fu. "Rapid circular microfluidic mixer utilizing unbalanced driving force." Biomedical Microdevices 9, no. 1 (2007): 43-50. https://doi.org/10.1007/s10544-006-9009-3

[30] Rahimi, Masoud, Neda Azimi, Mohammad Amin Parsamogadam, Alireza Rahimi, and Mohammad Moein Masahy. "Mixing performance of T, Y, and oriented Y-micromixers with spatially arranged outlet channel: evaluation with Villermaux/Dushman test reaction." Microsystem Technologies 23, no. 8 (2017): 3117-3130. https://doi.org/10.1007/s00542-016-3118-6

[31] Commenge, Jean-Marc, and Laurent Falk. "Villermaux-Dushman protocol for experimental characterization of micromixers." Chemical Engineering and Processing: Process Intensification 50, no. 10 (2011): 979-990. https://doi.org/10.1016/j.cep.2011.06.006 
[32] Pinot, Jordanna, J-M. Commenge, J-F. Portha, and Laurent Falk. "New protocol of the Villermaux-Dushman reaction system to characterize micromixing effect in viscous media." Chemical Engineering Science 118 (2014): 94-101. https://doi.org/10.1016/j.ces.2014.07.010

[33] Su, Jing, Minhua Cao, Ling Ren, and Changwen Hu. "Fe3O4-graphene nanocomposites with improved lithium storage and magnetism properties." The Journal of Physical Chemistry C 115, no. 30 (2011): 14469-14477. https://doi.org/10.1021/jp201666s

[34] Gray, Dodd, Adam McCaughan, and Bhaskar Mookerji. "Crystal structure of graphite, graphene and silicon." Physics for Solid State Applications 6 (2009).

[35] Georgakilas, Vasilios, Michal Otyepka, Athanasios B. Bourlinos, Vimlesh Chandra, Namdong Kim, K. Christian Kemp, Pavel Hobza, Radek Zboril, and Kwang S. Kim. "Functionalization of graphene: covalent and non-covalent approaches, derivatives and applications." Chemical reviews 112, no. 11 (2012): 6156-6214.

https://doi.org/10.1021/cr3000412

[36] Zhang, Ming, Danni Lei, Xiaoming Yin, Libao Chen, Qiuhong Li, Yanguo Wang, and Taihong Wang "Magnetite/graphene composites: microwave irradiation synthesis and enhanced cycling and rate performances for lithium ion batteries." Journal of Materials Chemistry 20, no. 26 (2010): 5538-5543. https://doi.org/10.1039/c0jm00638f

[37] Farghali, Mohamed A., Taher A. Salah El-Din, Abdullah M. Al-Enizi, and Ramadan M. El Bahnasawy. "Graphene/magnetite nanocomposite for potential environmental application." Int. J. Electrochem. Sci 10 (2015): 529-537.

[38] Meyer, J. C. "Transmission electron microscopy (TEM) of graphene." In Graphene, pp. 101-123. Woodhead Publishing, 2014. https://doi.org/10.1533/9780857099334.2.101

[39] Novoselov, Konstantin S., and A. K. Geim. "The rise of graphene." Nat. Mater 6, no. 3 (2007): 183-191.

[40] Liu, Panbo, Ying Huang, and Xiang Zhang. "Superparamagnetic Fe304 nanoparticles on graphene-polyaniline: Synthesis, characterization and their excellent electromagnetic absorption properties." Journal of alloys and compounds 596 (2014): 25-31.

https://doi.org/10.1016/j.jallcom.2014.01.188 